\title{
Enseñanza y aprendizaje de la matemática para informática empleando documentos con un formato computable (CDFs): una percepción docente en la Universidad Nacional de Costa Rica
}

\author{
Teaching and learning of mathematics for computer science using documents with a computable \\ format (CDFs): a teaching perception at the National University of Costa Rica \\ (1) Enrique Vílchez Quesada \\ enrique.vilchez.quesada@una.ac.cr \\ Universidad Nacional de Costa Rica \\ Costa Rica \\ Juan Félix Ávila Herrera \\ delagarita@gmail.com \\ Universidad Nacional de Costa Rica \\ Costa Rica
}

Resumen: La matemática básica entendida como un compendio de contenidos desarrollados en la educación secundaria, constituye un área de relevancia en la formación de cualquier futuro ingeniero. Esto es debido a que provee un andamiaje cognitivo necesario como un medio instrumental en materias vinculadas con carreras de ingeniería y particularmente, las de informática y ciencias de la computación. En el contexto de la carrera de Ingeniería en Sistemas de Información de la Universidad Nacional de Costa Rica (UNA), el rendimiento académico mostrado por la población estudiantil a este nivel devela importantes carencias conceptuales y algorítmicas. Ante este panorama, los autores del presente trabajo diseñaron ciento treinta aplicaciones para el estudio de: la lógica formal, la teoría de conjuntos, la teoría de funciones, los tópicos de inducción y progresiones, y la teoría de números. Se ha partido de la premisa de considerar la visualización matemática como un medio que facilita el tránsito de una percepción intuitiva al análisis, además de propiciar la comprensión conceptual y la construcción de conjeturas. El objetivo principal de este estudio residió en medir sobre un grupo de docentes de la Escuela de Matemática de la UNA, sus reacciones de significado y rescatar sus opiniones abiertas en cuanto a las ventajas, desventajas y oportunidades reales de uso sobre las aplicaciones CDFs diseñadas, como un recurso didáctico específicamente en la cátedra MAT030 Matemática para Informática que forma parte del currículo de la carrera Ingeniería en Sistemas de Información de esta institución de enseñanza superior. Las aplicaciones se programaron en el año 2019 en lenguaje Wolfram siendo publicadas en un formato de uso libre denominado CDF (Computable Document Format). Los resultados obtenidos con un enfoque cualitativo y cuantitativo se han centrado en rescatar las fortalezas, debilidades y recomendaciones sobre los CDFs creados para la cátedra MAT030.

Palabras Clave: CDFs, enseñanza, aprendizaje, visualización, matemática básica

Abstract: Basic mathematics understood as a compendium of contents developed in secondary education, constitutes an area of relevance in the training of any future engineer. This is since it provides a 
necessary cognitive scaffolding as an instrumental means in subjects related to engineering and particularly, informatics and computer science. In the context of the Information Systems Engineering degree at the National University of Costa Rica (UNA), the academic performance shown by the student population at this level reveals important conceptual and algorithmic deficiencies. Against this background, the authors of this work designed one hundred and thirty applications for the study of: formal logic, set theory, function theory, induction and progression topics, and number theory. It has been started from the premise of considering mathematical visualization as a means that facilitates the transition from an intuitive perception to analysis, in addition to promoting conceptual understanding and the construction of conjectures. The main objective of this study was to measure on a group of teachers of the School of Mathematics of the UNA, their reactions of meaning and rescue their open opinions regarding the advantages, disadvantages and real opportunities of use on the designed CDF applications, as a didactic resource specifically in the MAT030 Mathematics for Informatics chair that is part of the curriculum of the Information Systems Engineering career of this institution of higher education. The applications are programmed in the year 2019 in the Wolfram language and are published in a free-use format called CDF (Computable Document Format). The results obtained with a qualitative and quantitative approach have focused on rescuing the strengths, weaknesses and recommendations on the CDFs created for the MAT030 chair.

Keywords: CDFs, teaching, learning, visualization, basic mathematics

\section{Introducción}

En la Escuela de Informática de la Universidad Nacional de Costa Rica, desde hace algunos años, se ha venido evidenciando un bajo rendimiento académico en el primer curso de matemática que deben matricular los alumnos inscritos en la carrera de Ingeniería en Sistemas de Información. Por ejemplo, de acuerdo con la coordinación de cursos de servicio, para el I ciclo del año 2017, un 58\% de los estudiantes de nuevo ingreso fracasó, en el I ciclo del año 2018, un 75\% de los alumnos reprobó y para el I ciclo del año 2019 un 56 \% no terminó con éxito el curso. Esta materia denominada en el plan de estudios MAT030 Matemática para Informática, abarca cinco ejes temáticos principales: lógica proposicional, teoría de conjuntos, teoría de funciones, inducción y progresiones, y teoría de números. Como una respuesta proactiva ante esta problemática se formuló por parte de los autores, un proyecto de investigación en docencia titulado: CDFs como recurso didáctico para apoyar la materia MAT030, código institucional 0126-18, cuyo objetivo general consistió en analizar el impacto pedagógico y didáctico al utilizar un tipo especial de documento llamado CDF.

Las siglas en inglés CDF significan Computable Document Format, el cual es un tipo de archivo con un formato computable que permite al usuario no solo visualizar información, sino también, interactuar con ella mediante el empleo de diversos controladores, tales como: deslizadores, checkbox, barras de selectores, menús desplegables, botones, campos de texto, entre otros (Vílchez y Ávila, 2019). Los CDFs en general se desarrollan en un lenguaje de programación denominado Wolfram Language, que forma parte del conocido software comercial Wolfram Mathematica. La empresa Wolfram Research ha sido la encargada de poner a disposición de científicos y educadores, esta nueva forma estandarizada de cómputo de datos y conocimiento. Esta opción es tan estándar como un PDF (Portable Document Format), pero tan dinámica como un programa de cómputo.

A raíz de las tareas vinculadas con el proyecto en docencia antes citado se generaron en el año 2019 por parte de los autores, dos importantes recursos didácticos para ejercer la docencia en la materia MAT030, a saber: un libro de texto con teoría y ejercicios y ciento treinta aplicaciones CDFs empleadas en la obra, bajo la consigna de abordar los contenidos con un enfoque asistido por computadora. Esta opción didáctica reconoce lo esencial de la visualización en la enseñanza y el aprendizaje de la matemática, siendo ésta, tal y como lo señala Godino, et al. (2012) un medio fortificador en la reali- 
zación de operaciones mentales que transforman un objeto visual hacia la búsqueda de argumentos de naturaleza analítica y abstracta.

Como una primera etapa de implementación, el libro de texto titulado Matemática para Informática mediante el uso de CDFs y los documentos con un formato computable diseñados, se sometieron a escrutinio por medio de un taller de divulgación dirigido a los profesores de la Escuela de Matemática de la UNA. Al finalizar esta actividad, se les aplicó a los docentes participantes un diferencial semántico con el objetivo de medir sus reacciones de significado ante los materiales creados dando además cabida, a sus opiniones abiertas en cuanto a las ventajas, desventajas y oportunidades reales de uso. El presente trabajo comparte los hallazgos más significativos alcanzados en este contexto.

\section{Enseñanza y aprendizaje de la matemática básica a nivel superior}

La matemática como disciplina científica está típicamente catalogada como un área de difícil comprensión, exclusiva para aquellos estudiantes que manifiestan extraordinarios talentos intelectuales. Estas presunciones son normalmente atribuibles a la naturaleza abstracta de los contenidos que se abordan, sin embargo, históricamente, dichas posturas de corte positivista han ignorado la influencia preponderante que los docentes en las aulas universitarias pueden tener para favorecer una mejoría en lo denominado por Gamboa (2016) como "dominio afectivo y emocional" (p. 64).

La ansiedad que enfrentan los alumnos recién llegados a los recintos universitarios constituye una de las razones principales del fracaso escolar mostrado en cursos de matemática introductoria. Al respecto, Espinoza y Delgado (2016) señalan: "se han llevado a cabo investigaciones sobre rendimiento y ansiedad matemática en todos los niveles, desde secundaria hasta el nivel universitario y se ha encontrado que existe una relación firme y evidente entre rendimiento y ansiedad matemática" (p. 210).

En las instituciones de enseñanza superior estos resultados científicos parecen permanecer desapercibidos. La universidad se ha convertido en un instrumento de selección natural donde prevalece "el más fuerte" y sucumbe el menos favorecido, siendo responsabilidad intrínseca del sistema educativo, tal y como lo señala Cantoral, crear los mecanismos necesarios que permitan a cualquier alumno retomar su proceso de formación académica independientemente de sus condiciones particulares (2001).

Los conocimientos previos de los estudiantes de primer ingreso en la universidad, constituye otro tema de importancia al analizar los procesos de enseñanza y aprendizaje de la matemática en cursos propedéuticos. Es innegable, como estos conocimientos resultan ser fundamentales para poder garantizar el éxito futuro de los alumnos en el estudio del precálculo u otras áreas esenciales como la lógica y la teoría de conjuntos. En esta dirección, Mota y Valles afirman:

Es evidente la gran importancia que tienen los conocimientos previos en la adquisición de nuevos conocimientos. En el caso del área de las matemáticas a nivel universitario, se hace imprescindible que el estudiante tenga sólidas bases sobre el conocimiento matemático de secundaria para poder aprehender con éxito los nuevos saberes matemáticos universitarios, esto debido a que esos conocimientos de base sirven de 'anclaje' para los nuevos saberes; no obstante, las numerosas investigaciones (...), dejan al descubierto la ruptura existente entre esos dos conocimientos (Mota y Valles, 2015, p. 89).

Aunado a lo anterior, las diferencias formativas de la población estudiantil diversificada que normalmente ingresa a las instituciones universitarias ponen de manifiesto, un escenario aún más complejo. En Costa Rica, por ejemplo, existen claras desigualdades en el rendimiento académico de los alumnos con respecto a la prueba nacional de educación media en matemática, dependiendo de factores, tales como el geográfico y el socioeconómico. Según Castillo, Chavarría y García, los colegios en el área metropolitana presentan usualmente un mejor rendimiento en comparación con los de zonas rurales y, asimismo, los centros educativos privados muestran un nivel de desempeño más favorable (2016).

Enseñanza y aprendizaje de la matemática para informática empleando documentos con un formato computable (CDFs): una percepción docente en la Universidad Nacional de Costa Rica. Vúlchez, E.; Ávila, J.

Derechos Reservados (c) 2021 Revista digital Matemática, Educación e Internet (https://tecdigital.tec.ac.cr/revistamatematica/) 
Frente a estos retos sustanciales, los autores del presente trabajo han concebido en el uso de la tecnología educativa, un medio crucial, que bien utilizado, crea viaductos conciliadores entre la ansiedad matemática y las malas bases conceptuales o procedimentales de los estudiantes, con oportunidades de crecimiento académico. Có, et al., opinan que las herramientas computaciones en este sentido, pueden convertirse en herramientas cognitivas dentro del marco de una planificación didáctica apropiada de actividades y elaboración de materiales (2011).

Se ha partido de este enfoque, con miras a transformar el modelo tradicional de enseñanza y aprendizaje imperante en las prácticas pedagógicas de la materia MAT030 en la Universidad Nacional de Costa Rica, facilitando el uso de documentos con un formato computable con el objetivo de desarrollar competencias matemáticas, como las adoptadas por Rico: razonamiento, argumentación, modelación, planteo y resolución de problemas, representación y empleo del lenguaje formal (2006).

El uso de las tecnologías con fines educativos ofrece una alternativa metodológica refrescante, pues: "abren un espacio en el que los estudiantes pueden manipular de manera directa los objetos matemáticos y sus relaciones" (Cruz y Puentes, 2012, p. 16). En la cátedra del curso MAT030 los CDFs diseñados conforman un compendio de recursos de mediación que amplían las nociones teórico-prácticas convencionales con objetos visuales, donde es posible experimentar variaciones de registros a través de la modificación de los parámetros que los caracterizan. En este plano, la visualización matemática cobra una importancia significativa.

\section{Visualización en matemática}

La visualización en matemática es una categoría especial de pensamiento que adecuadamente canalizado, puede contribuir con la construcción de estructuras de conocimiento abstracto. En ella, de acuerdo con Marmolejo, las representaciones visuales suponen la generación de una serie de registros que actúan como puentes, facilitando la asimilación de conceptos y propiedades y, aún más relevante, la formulación de relaciones, razonamientos y conjeturas (2010).

La visualización se considera una actividad cognitiva que abarca un rango de acción superior al de la visión convencional. Duval, citado por Godino, et al. (2012), señala una clara distinción entre visión y visualización, siendo la primera una simple percepción de un individuo sobre lo que observa y la segunda una representación más global, que expresa la configuración interna de un objeto traducida en las relaciones explícitas o implícitas de las componentes que lo caracterizan. Por lo anterior, la visualización en matemática es una tarea compleja, pues demanda del alumno una capacidad de selección asertiva, permitiéndole inferir los atributos visuales primarios.

La utilización de programas computacionales especializados en la enseñanza y el aprendizaje de la matemática, bajo esta perspectiva, ofrece alternativas viables que asisten en el desarrollo de habilidades de interpretación, análisis y aprehensión, basadas en la visualización. Además, en paralelo, tal y como lo señala Macias, mediante una manipulación dinámica de los registros facilitados por un software, los estudiantes pueden descubrir en sus propias ideas, sólidos contenidos matemáticos formales (2007).

Los CDFs creados para la materia MAT030, en esta línea de reflexión, proveen herramientas de cálculo, ensayo y error, escenarios experimentales, demostraciones y recursos de ejercitación algorítmica, tomando como punto de partida la visualización matemática en un ámbito didáctico, donde se promueven estrategias de aprendizaje bajo una metodología instruccional constructivista. Cuicas, et al. (2007), vislumbran en el empleo de software en la educación matemática, un elemento clave para el logro de un aprendizaje más significativo, estos autores mencionan: "con el uso del software la atención se enfoca en facilitar que el discente aprenda a procesar la información de la materia, así como, en la transferencia y generalización de los aprendizajes a otros aspectos académicos o no" (p. 7). 
Los autores de esta propuesta han apostado en el diseño de documentos con un formato computable, la transformación pedagógica necesaria que desde hace algunos años demandan los estudiantes de la cátedra del curso MAT030.

\section{CDFs: documentos con un formato computable}

Los documentos con un formato computable (CDFs) son un tipo de archivo propietario del conocido software comercial Wolfram Mathematica.

La empresa Wolfram Research ha puesto al servicio de la comunidad académica este importante formato, con la principal intención de facilitar un estándar de documentos, tan comunes como un PDF, pero tan interactivos como un programa de computadora (Wolfram, 2019).

Los CDFs se desarrollan en lenguaje Wolfram, sin embargo, su uso solamente está supeditado a la instalación de un plug in que la misma empresa distribuye: Wolfram CDF Player, existiendo, además, una versión gratuita. El plug in está disponible para distintos sistemas operativos como Windows, Linux y Mac.

Los CDFs tienen una amplia gama de aplicaciones, bajo una óptica computacional, desde la elaboración de un informe técnico, hasta el diseño de la simulación de un fenómeno físico, "prácticamente todo lo que es posible desarrollar en un cuaderno convencional de Mathematica, será un recurso fácil de exportar como un CDF" (Vílchez y Ávila, 2019, p. 2). En un contexto educativo, los CDFs pueden convertirse en beneficiosas herramientas para mediar una metodología en la que se logre combinar de forma exitosa los contenidos matemáticos abstractos y los procesos heurísticos que conduzcan en el mejor de los casos, al aprendizaje, guiado por la intuición, los conocimientos previos de los alumnos y la visualización matemática, como motores que estimulan la incubación de nuevas conexiones cognitivas.

\section{CDFs para la enseñanza y el aprendizaje de la materia: MAT030 Ma- temática para Informática}

Los autores de la presente propuesta como ya se señaló, diseñaron una serie de documentos con un formato computable que fueron publicados en dos versiones: una orientada a su uso en un ordenador y la otra, mediante el empleo de dispositivos móviles. Las aplicaciones tienen una concordancia directa con el programa curricular del curso MAT030 Matemática para Informática, pues pretenden brindar otra opción metodológica a los profesores y estudiantes pertenecientes a esta asignatura integrada en el plan de estudios de la carrera Ingeniería en Sistemas de Información de la UNA.

Los CDFs se encuentran disponibles para su libre descarga, en la dirección URL: https : //www . escinf . una.ac.cr/CDF/index.php/matematica-para-informatica. En correspondencia con lo planteado en las secciones 2, 3 y 4, estas aplicaciones fueron concebidas como un conjunto de recursos didácticos donde prevalece la interacción con objetos visuales a través de la modificación de sus registros. Estas tareas cognitivas, procuran que la población estudiantil logre alcanzar la adquisición de competencias matemáticas, una mejor profundización conceptual y procedimental y, en general, un aprendizaje más significativo de los contenidos de la materia MAT030.

Se compartirá en esta sección, a manera de ejemplo, un CDF creado con el objetivo de estudiar el concepto de función biyectiva. En la figura 1, se muestra el CDF correspondiente. Al arrastrar el deslizador

Enseñanza y aprendizaje de la matemática para informática empleando documentos con un formato computable (CDFs): una percepción docente en la Universidad Nacional de Costa Rica. Vílchez, E.; Ávila, J.

Derechos Reservados @ 2021 Revista digital Matemática, Educación e Internet (https://tecdigital.tec.ac.cr/revistamatematica/) 
"No de pares ordenados" se amplía o reduce el tamaño de una tabla de valores donde se representa una relación. Se espera que el estudiante analice si esta relación es una función y si lo es, si satisface la definición de inyectividad, sobreyectividad y biyectividad. Al marcarse el check "Solución", se suministra una retroalimentación con la respuesta del ejercicio.

Los ejemplos generados por el CDF son pseudoaleatorios, por lo que, cada vez que se presiona el botón "Generar una nueva tabla", la aplicación exhibe un ejercicio distinto.

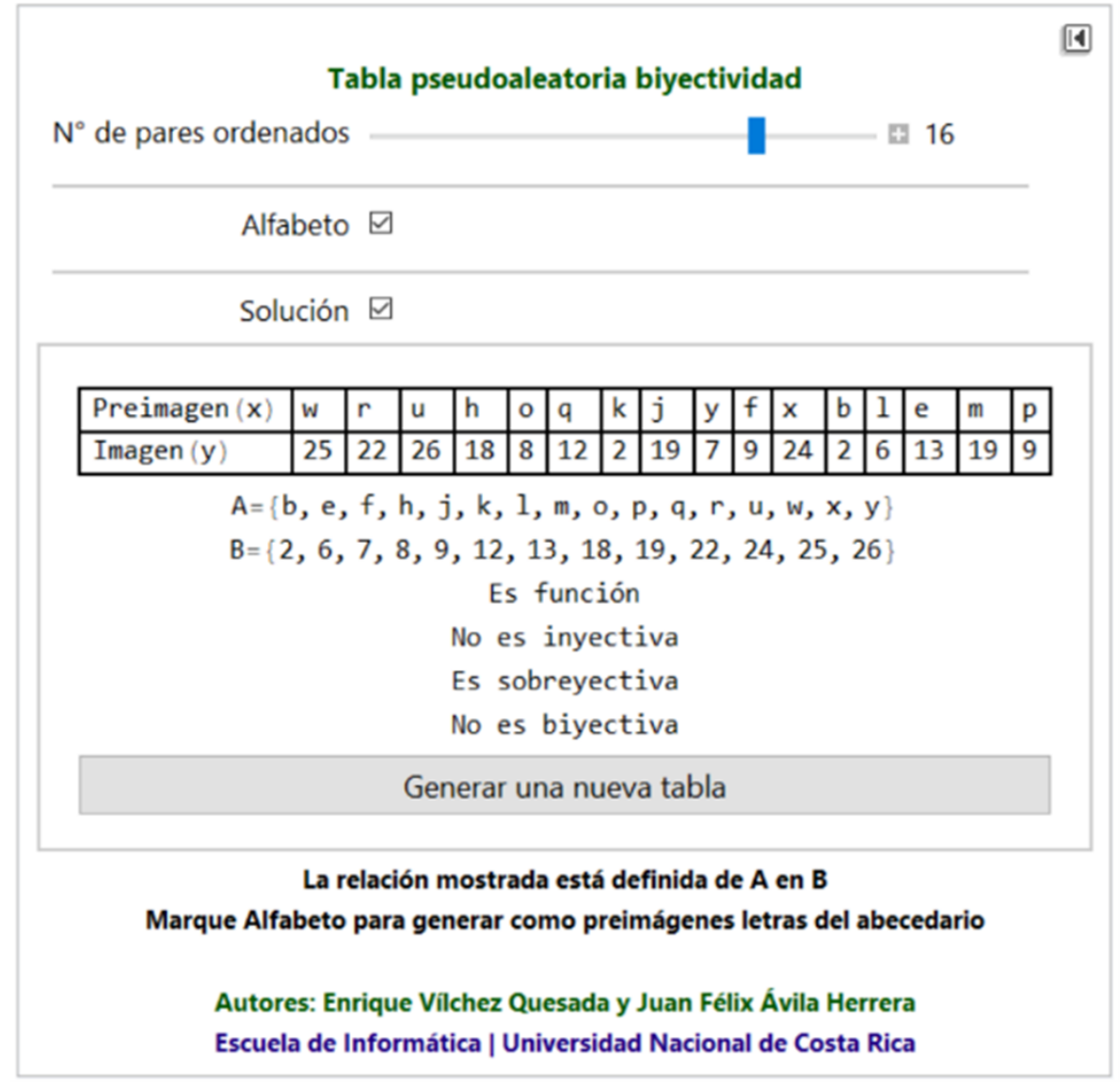

Figura 1: CDF “Tabla pseudoaleatoria biyectividad”. Fuente: Elaboración propia.

Se insta al lector a explorar otros de los CDFs publicados en: https://www.escinf.una.ac.cr/CDF/ index.php/matematica-para-informatica.

\section{Implementación de uso de los CDFs: percepción docente}

En el segundo semestre del año 2019 se efectuó un proceso de validación con un grupo de dieciséis docentes participantes, en su mayoría, miembros de la cátedra del curso MAT030 Matemática para Informática. A esta muestra, se les compartió dos tipos de recursos didácticos diseñados por los autores, mediante un taller introductorio que tuvo una duración de tres horas (la sesión de trabajo fue grabada y puede consultarse en: https : //youtu . be/aZaHB_bbSDQ). Los profesores usaron en la actividad el libro denominado Matemática para Informática mediante el uso de CDFs y veinte aplicaciones CDFs seleccionadas de forma representativa para brindar una idea global sobre las potencialidades de los documentos con un formato computable programados.

Al finalizar el taller, se les solicitó a los participantes completar un instrumento caracterizado por uti-

Enseñanza y aprendizaje de la matemática para informática empleando documentos con un formato computable (CDFs): una percepción docente en la Universidad Nacional de Costa Rica. Vílchez, E.; Ávila, J.

Derechos Reservados @ 2021 Revista digital Matemática, Educación e Internet (https://tecdigital.tec.ac.cr/revistamatematica/) 
lizar la técnica de diferencial semántico y algunas preguntas abiertas. En el instrumento se añadieron dieciséis adjetivos bipolares basados en otro diferencial semántico elaborado por Meza y Azofeifa (2010), quiénes analizaron su nivel de fiabilidad a través de la consulta con expertos y el uso del coeficiente Alfa de Cronbach. Se tomó la decisión de usar este tipo de mecanismo de recolección de información por el objetivo de naturaleza afectiva y de opinión en cuanto a las fortalezas y debilidades del texto y los CDFs como herramientas didácticas para impartir la materia MAT030.

Los adjetivos se cuantificaron en una escala del 1 al 7 tabulados en el programa estadístico SPSS, siendo 1 un valor que indica una postura muy negativa y 7 una actitud muy positiva. El análisis e interpretación de los datos se sustentó en el cálculo de la media aritmética p para cada uno de los adjetivos bipolares, según las siguientes categorías: $1 \leq p<2$ percepción muy negativa, $2 \leq p<3$ moderadamente negativa, $3 \leq p<4$ negativa baja, $4 \leq p<5$ positiva baja, $5 \leq p<6$ positiva moderada y $6 \leq p \leq 7$ percepción muy positiva.

En la tabla 1 se describen los resultados obtenidos sobre la percepción de los CDFs compartidos con la muestra.

Tabla 1: El uso de los CDFs como un recurso para la enseñanza y el aprendizaje.

\begin{tabular}{lccl}
\hline Adjetivos bipolares & Media & Desviación estándar & Interpretación \\
\hline Inútil-Útil & 6.625 & 0.61914 & Muy positiva \\
Difícil-Fácil & 5.875 & 0.88506 & Positiva moderada \\
Aburrido-Divertido & 5.75 & 0.7746 & Positiva moderada \\
Confuso-Claro & 5.75 & 1 & Positiva moderada \\
Frustrante-Motivador & 6.3125 & 0.70415 & Muy positiva \\
Estresante-Relajante & 5.75 & 0.85635 & Positiva moderada \\
Complicado-Sencillo & 5.8125 & 0.91059 & Positiva moderada \\
Innecesario-Necesario & 5.9375 & 0.92871 & Positiva moderada \\
Desagradable-Agradable & 6.1875 & 0.75 & Muy positiva \\
Irrelevante-Relevante & 6.125 & 1.08781 & Muy positiva \\
No formativo-Formativo & 6.625 & 0.7188 & Muy positiva \\
No entendible-Entendible & 6.3125 & 0.70415 & Muy positiva \\
Inaplicable-Aplicable & 6.5625 & 0.51235 & Muy positiva \\
Inadecuado-Adecuado & 6.4375 & 0.81394 & Muy positiva \\
Incompleto-Completo & 5.875 & 0.88506 & Positiva moderada \\
Negativo-Positivo & 6.5 & 0.63246 & Muy positiva \\
\hline
\end{tabular}

Fuente: Elaboración propia.

Se destaca en la tabla anterior como todas las interpretaciones se encuentran únicamente en el rango de "muy positivas" y "positivas moderadas". Los docentes conciben con una actitud extrema positiva el uso de los documentos con un formato computable para la enseñanza y el aprendizaje, en los siguientes aspectos de empleo: útiles, motivadores, agradables, relevantes, formativos, entendibles, aplicables, adecuados y positivos. De forma positiva moderada perciben las aplicaciones CDFs como un recurso: fácil, divertido, claro, relajante, sencillo, necesario y completo. Algunos profesores indicaron en las preguntas abiertas del instrumento que su utilización podría entrabarse en la población estudiantil, al tener que recurrir a ciertas instrucciones propias del software Wolfram Mathematica, por otra parte, también otros mencionaron el requerimiento de aumentar el nivel cognitivo de los recursos didácticos con la finalidad de igualarse en complejidad, al compararlos con algunos de los ejes temáticos y enfoques adoptados por la cátedra MAT030.

Con respecto a la valoración del libro de texto Matemática para Informática mediante el uso de CDFs, la tabla 2 resume las percepciones encontradas, todas ellas bajo la interpretación "muy positivas". Los 
docentes opinaron que el texto es: útil, claro, adecuado, formativo y relevante.

Tabla 2: Libro de texto Matemática para Informática mediante el uso de CDFs.

\begin{tabular}{lccl}
\hline Adjetivos bipolares & Media & Desviación estándar & Interpretación \\
\hline Inútil-Útil & 6.1875 & 1.04682 & Muy positiva \\
Confuso-Claro & 6.4375 & 0.72744 & Muy positiva \\
Inadecuado-Adecuado & 6.25 & 0.85635 & Muy positiva \\
No formativo-Formativo & 6.4375 & 0.51235 & Muy positiva \\
Irrelevante-Relevante & 6.4375 & 0.72744 & Muy positiva \\
\hline
\end{tabular}

Fuente: Elaboración propia.

En el instrumento se incluyeron, además, dos preguntas abiertas para rescatar posibles fortalezas y debilidades del libro y los CDFs diseñados: ¿señale al menos tres fortalezas que considere pertinentes del libro de texto y/o los CDFs? y ¿señale al menos tres debilidades que considere pertinentes del libro de texto y/o los CDFs? Estas se resumen en las opiniones sistematizadas por los autores expuestas en la tabla 3.

Tabla 3: Fortalezas y debilidades del libro y los CDFs.

\begin{tabular}{l} 
Fortalezas \\
\hline - Apoyan la comprensión conceptual de los \\
temas del curso. Se incentiva la visualización \\
de conceptos y una práctica más interactiva. \\
- Contribuyen con la formación autodidacta \\
del alumno en contenidos teóricos y prácti- \\
cos. Por ejemplo, en la revisión y generación \\
automática de ejercicios, sin perder la profun- \\
didad de análisis. \\
- Fomentan la autoevaluación del estudiante \\
y un aumento de su motivación hacia la ma- \\
teria.
\end{tabular}

- El formato de los recursos es agradable (uso de color, íconos, estructura, imágenes de referencia y diseño gráfico en general) facilitando que el alumno concentre su atención en los puntos relevantes y mejore su disposición.

- Los recursos son interactivos (multimediales con videos y aplicaciones), innovadores y pertinentes, incorporando actividades complementarias de aula.

- Es un material accesible, gratuito y portable tanto para el alumno como para el profesor.

- Pueden simplificar la labor docente en un avance más efectivo de los contenidos en clase, dedicando más tiempo a las explicaciones y no al tradicional hábito de transcripción.

\section{Debilidades}

- Los temas se presentan con un nivel más básico de lo que usualmente se maneja en la cátedra.

- Las herramientas de software pueden ser empleadas por los alumnos para no trabajar y cometer fraude en las evaluaciones del curso.

- No es un material accesible para estudiantes con discapacidad visual.

- Algunos CDFs no dan una retroalimentación adecuada cuando las respuestas del estudiante son incorrectas.

- Se requiere el uso de Internet y en algunos CDFs el empleo del software Wolfram Mathematica (que no es de uso libre).

- Algunos CDFs son difíciles de entender. 
Una de las principales preocupaciones de la población docente en este taller, residió en considerar las aplicaciones CDFs como una amenaza ante posibles actos de fraude por parte del estudiantado. Esta preocupación es muy válida al circunscribirse el empleo de estos recursos bajo un esquema de evaluación tradicional. Desde este punto de vista, consideramos importante reconocer que, en iniciativas de uso de software con fines didácticos, la evaluación debe transformarse en concordancia con un ambiente de aprendizaje experimental y no algorítmico.

En cuanto a la implementación de los CDFs los autores lograron identificar aplicaciones puntuales donde se hace necesario mejorar la manera en cómo se le presenta a un alumno una respuesta incorrecta, incitándolo más a descubrir por su propia cuenta errores conceptuales o de procedimiento. También, se seleccionaron ciertos CDFs cuyas instrucciones resultaron ser difusas para sus potenciales usuarios, con el objetivo de mejorarlas pensando en la población estudiantil.

En cuanto a la posibilidad de empleo real del libro y los CDFs en la labor docente, quince colegas estuvieron anuentes a utilizar los recursos y solamente un profesor indicó no saber, al considerarlo una decisión de toda la cátedra. Sí señalaron la necesidad de balancear el enfoque tradicional característico de la materia MAT030 con el asistido por computadora.

\section{Conclusiones}

La experiencia de implementación de uso del libro de texto Matemática para Informática mediante el uso de CDFs y las aplicaciones CDFs elaboradas para el curso MAT030, arrojó resultados preliminares bastante positivos. Los recursos didácticos se muestran ante la población docente participante como: interactivos, innovadores, accesibles, portables, útiles, motivadores, agradables, relevantes, formativos, entendibles, aplicables, pertinentes y positivos. Adicionalmente, hay un panorama favorable a nivel general sobre su posibilidad de uso real en el contexto de esta cátedra.

Como posibles actividades de mejora corresponderá a los autores de esta propuesta aumentar el nivel de dificultad en algunas de las secciones del libro con la intención de adecuarse más adecuadamente a las necesidades del curso MAT030. Además de ello, queda abierto un espacio de mejora en algunas de las aplicaciones CDFs respecto a su claridad y al hecho de proporcionar un feedback más apropiado para la población estudiantil, cuando han respondido de una forma incorrecta en los documentos con un formato computable.

\section{Referencias}

[1] Cantoral, R. (2001). Enseñanza de la matemática en la educación superior. Revista Electrónica Sinéctica, 1(19), 3-27. Recuperado de https://www. redalyc.org/pdf/998/ 99817935002.pdf

[2] Castillo, M., Chavarría, J. y García, M. (2016). Rendimiento académico en las pruebas nacionales de matemática en colegios del área metropolitana y zonas alejadas de Costa Rica en 2013. Revista UNICIENCIA, 30(1), 85-97. doi: http://dx. doi.org/10.15359/ ru. $30-1.5$

[3] Có, P., Del Sastre, M., Panella, E. y Sadagorsky, A. (2011). Valoración del impacto de los software matemáticos en el aprendizaje y la enseñanza de la matemática básica en carreras de ingeniería. En P. Lestón (Ed.), Acta Latinoamericana de Matemática Educativa 24 (RELME 24, pp. 1134-1141). Congreso realizado en Guatemala, Comité La- 
tinoamericano de Matemática Educativa. Recuperado de https://www.clame.org.mx/ documentos/alme24.pdf

[4] Cruz, I. y Puentes, A. (2012). Innovación Educativa: Uso de las TIC en la enseñanza de la Matemática Básica. Edmetic: Revista de Educación Mediática y TIC, 1(2), 127144. Recuperado de https://www. uco.es/ucopress/ojs/index.php/edmetic/article/ view/2855/2743

[5] Cuicas, M., Debel, E., Casadei, L. y Alvarez, Z. (2007). El software matemático como herramienta para el desarrollo de habilidades del pensamiento y mejoramiento del aprendizaje de las matemáticas. Revista Electrónica Actualidades Investigativas en Educación, 7(2), 1-34. Recuperado de https : //www. redalyc.org/pdf/447/44770209. pdf

[6] Espinoza, J. y Delgado, I. (2016). Ansiedad matemática en estudiantes de primer año de la Universidad Nacional de Costa Rica. Revista Universidad en Diálogo, 5(2), 207-217. Recuperado de https://www. revistas.una.ac.cr/index.php/dialogo/article/view/ 8442

[7] Gamboa, R. (2016). ¿Es necesario profundizar en la relación entre docente de matemáticas y la formación de las actitudes y creencias hacia la disciplina? Revista UNICIENCIA, 30(1), 57-84. doi: https : //doi. org/10. 15359/ru. 30-1.4

[8] Godino, J., Gonzato, M., Cajaraville, J. y Fernández, T. (2012). Una aproximación ontosemiótica a la visualización en educación matemática. Enseñanza de las Ciencias: Revista de Investigación y Experiencias Didácticas, 30(2), 109-130. Recuperado de https: //www. raco.cat/index.php/Ensenanza/article/view/254506/391053

[9] Macias, D. (2007). Las nuevas tecnologías y el aprendizaje de las matemáticas. Revista Iberoamericana de Educación, 42(4), 1-17. doi: https://rieoei.org/historico/ deloslectores/1517Macias.pdf

[10] Marmolejo, G. (2010). La visualización en los primeros ciclos de la educación básica. Posibilidades y complejidad. Revista Sigma, 10(2), 10-26. Recuperado de https: //dialnet.unirioja.es/descarga/articulo/3714823.pdf

[11] Meza, L. y Azofeifa, R. (2010). Actitud hacia la matemática de las y los estudiantes del curso Matemática General en el ITCR: análisis general y por género. En J. Ávila (Ed.), II Congreso Internacional Computación y Matemática. Congreso realizado en Costa Rica, Universidad Nacional de Costa Rica.

[12] Mota, D. y Valles, R. (2015). Papel de los conocimientos previos en el aprendizaje de la matemática universitaria. Revista Acta Scientiarum, 37(1), 85-90. doi: https ://doi. org/10.4025/actascieduc.v37i1.21040

[13] Rico, L. (2006). La competencia matemática en PISA. PNA: Revista de Investigación en Didáctica de la Matemática, 1(2), 47-66. Recuperado de http://digibug.ugr.es/ handle/10481/4703

[14] Vílchez, E. y Ávila, J. (2019). Desarrollo de documentos con un formato computable utilizando el software Wolfram Mathematica. Revista digital Matemática, Educación e Internet, 20(1), 1-29. Recuperado de https://tecdigital.tec.ac.cr/ revistamatematica/Secciones/Didactica_y_Software/RevistaDigital_FAvila_V20_ n1_2019/index.html

[15] Wolfram. (2019). Computable Document Format. USA: Wolfram. Recuperado de https: //www. wolfram. com/cdf 